\title{
DFT Investigation of the Structual and Optoelectronic Properties of Alkali Metal Hydrides $\mathrm{MH}(\mathrm{M}=\mathrm{Li}, \mathrm{Na})$
}

\author{
Taieb Iliass \\ Materials Science and Informatics Laboratory \\ Faculty of Science, University of Djelfa \\ Djelfa, Algeria \\ iliasstaieb@yahoo.com \\ Ahmed Gueddim \\ Materials Science and Informatics Laboratory \\ Faculty of Science, University of Djelfa \\ Djelfa, Algeria \\ ahmed_gueddim@yahoo.fr
}

\author{
Hamza Ziani \\ Materials Science and Informatics Laboratory \\ Faculty of Science, University of Djelfa \\ Djelfa, Algeria \\ hamza_ziani@yahoo.com \\ A. Djamel Guibadj \\ Laboratory of Physical Chemistry of Materials \\ Faculty of Science, University of Laghouat \\ Laghouat, Algeria \\ d.guibadj@gmail.com
}

\begin{abstract}
This paper presents ab initio calculations within the Density Functional Theory (DFT) for the structural and optoelectronic properties of the alkali metal hydrides $\mathrm{LiH}$ and $\mathrm{NaH}$ in rocksalt structure (B1). This study used the Generalized Gradient Approximation (GGA) of Wu-Cohen to consider the electronic exchange and correlation interactions. In addition, the Tran-Blaha modified Becke-Johnson exchange potential was used with the GGA approach (GGA-TBmBJ) to calculate the band structure with high accuracy. The structural properties, namely the lattice parameter, the bulk modulus, and the pressure derivative of the bulk modulus were determined and found to be generally in good agreement with other research findings. Furthermore, the energy band gaps, the Density Of States (DOS), the static and high-frequency dielectric constant, along the refractive index were addressed and analyzed. These results could be useful for hydrogen storage purposes.
\end{abstract}

\section{Keywords-Hydrogen storage; LiH; $\mathrm{NaH}$; ab initio; mBJ-GGA}

\section{INTRODUCTION}

The energy transition is necessary in order to meet the increasing energy needs and cope with global warming. The main drivers for this transition are the adoption of responsible consumer practices and the increased use of renewable sources of energy. This transition also requires efficient processes for production, conversion, storage, and transport of energy. Many scientific and technological challenges exist in each of those axes. As the problem of suitable materials is ubiquitous, materials science is at the heart of this research. The challenge is to obtain and optimize materials having specific properties to fulfill the requirements of renewable energy.

Hydrogen is a transportable, storable, and convertible energy medium and has great potential to become the ultimate solution that combines energy security, resource availability, and environmental compatibility. However, the greatest challenge in the development of hydrogen-based technologies is how to store hydrogen safely, effectively, and economically [1-5]. Depending on the physical state of hydrogen, three different routes are currently used for its storage: compressed gas, liquid, and solid-state. Hydrogen stored as a solid-state material has potential advantages in terms of volumetric density and safety, compared to compressed gas or liquid [611]. In the case of solid-state, the bonds between hydrogen and the host material can be of different kinds and strengths. Both van der Waals weak and chemisorptive strong bonds can be involved [11]. It is possible to increase the density of hydrogen, as it is packed with $\mathrm{H}-\mathrm{H}$ distances up to $170 \mathrm{~kg} / \mathrm{m}^{3}$ in many hydride-type materials. This value is higher than the density of hydrogen in a liquid state [12]. Numerous studies have been conducted to determine and design the most suitable materials with convenient gravimetric and volumetric densities for hydrogen storage. These materials span a large class, from conventional metal hydrides to complex hydrides, microporous materials, and clathrate hydrates. This study addresses some fundamental properties of $\mathrm{LiH}$ and $\mathrm{NaH}$ using the first principles calculations to utilize the Density Functional Theory (DFT). Such calculations are of great interest before the synthesis procedure, which is a very time-consuming effort. The structural properties along with their electronic and optical features are explored and analyzed for the materials under load in the rock-salt (B1) structure. The comparison with experimental and theoretical data, when possible, shows satisfactory agreement in general.

\section{COMPUTATIONAL METHODOLOGY}

This study used the Full Potential Linearized Augmented Plane Wave (FP-LAPW) method, based on the DFT [13] that constitutes the core of the WIEN2k code [14]. Exchangecorrelation energy was calculated using the WC-GGA [15] when dealing with structural properties. The exchangecorrelation functional $\mathrm{mBJ}$ [16] was applied to determine the electronic and other optical characteristics. In the FP-LAPW 
method, the wave function and the potential are developed as sums of spherical harmonic functions inside non-overlapping spheres, surrounding the atomic sites (muffin-tin spheres), and a set of plane waves in the interstitial region. A plane wave cutoff of $R_{M T}$. kmax $=7$ (where $R_{M T}$ is the smallest muffin-tin radius in the unit cell) was used. $\mathrm{R}_{\mathrm{MT}}$ 's were chosen to be 1.7, 2.1, and 2.5a.u. for $\mathrm{H}, \mathrm{Li}$, and $\mathrm{Na}$, respectively. The k-sampling over the Brillouin zone was performed up to a $13 \times 13 \times 13$ MonkorstPack [17-20] mesh for both considered materials. The number of k-points and the plane wave cut-off energy varied in such a manner as to obtain total energy convergence. The convergence of these self-consistent calculations was considered to be obtained within an error of $10^{-5} \mathrm{Ry}$ on the total energy of the crystals studied.

\section{RESULTS AND DISCUSSION}

The total energy was calculated from the WC-GGA approach as a function of the volume of $\mathrm{LiH}$ and $\mathrm{NaH}$ in the rocksalt structure. At first, the total energy for some selected lattice parameter values was calculated. The results obtained were then written according to Murnaghan's equation of state [21]. This allowed determining the equilibrium lattice parameter $a_{0}$, the zero pressure bulk modulus $B_{0}$, and the firstorder pressure derivative of the bulk modulus $B^{\prime}$ for the ground-state. These results are summarized in Table I with experimental and theoretical data from other researches for comparison. The WC-GGA lattice parameter in this study is generally closer to the experiment than those of the GGA or LDA approaches [27-29]. The $a_{0}$, calculated for both $\mathrm{LiH}$ and $\mathrm{NaH}$ from WC-GGA, agrees quite well with those reported in $[22,23]$. The same remark can be drawn for the bulk modulus $B_{0}$, which agrees within a few percent with other experimental or theoretical values [22, 24-26].

TABLE I. $\quad a_{0}, B_{0}$, AND $B^{\prime}$ FOR LiH AND NaH IN B1 STRUCTURE USING GGA AND WC-GGA ALONG WITH THE VALUES REPORTED IN THE LITERATURE.

\begin{tabular}{|c|c|c|c|}
\hline Material & $a_{0}(\AA)$ & $B_{0}$ (GPa) & $B^{\prime}$ \\
\hline LiH & $\begin{array}{c}3.9934^{\mathrm{a}} \\
4.0153^{\mathrm{b}} \\
4.012^{\mathrm{c}} \\
3.92^{\mathrm{d}}\end{array}$ & $\begin{array}{c}38.1840^{\mathrm{a}} \\
41.1635^{\mathrm{b}} \\
36.228^{\mathrm{c}} \\
36.60^{\mathrm{e}} \\
34.7^{\mathrm{f}} \\
\end{array}$ & $\begin{array}{c}3.9913^{\mathrm{a}} \\
3.0976^{\mathrm{b}} \\
3.51^{\mathrm{c}} \\
3.40^{\mathrm{e}}\end{array}$ \\
\hline $\mathrm{NaH}$ & $\begin{array}{c}4.8051^{\mathrm{a}} \\
4.8347^{\mathrm{b}} \\
4.833^{\mathrm{c}} \\
4.775^{\mathrm{d}}\end{array}$ & $\begin{array}{c}25.9068^{\mathrm{a}} \\
23.0855^{\mathrm{b}} \\
23.546^{\mathrm{c}} \\
19.40^{\mathrm{f}} \\
22.9^{\mathrm{g}}\end{array}$ & $\begin{array}{c}4.1001^{\mathrm{a}} \\
6.560^{\mathrm{b}} \\
3.90^{\mathrm{c}} \\
4.40^{\mathrm{f}}\end{array}$ \\
\hline
\end{tabular}

c. [22], d. [23], e. [24, f. Experimental value quoted in [25], g. [26]

The formation energy is an important thermodynamic quantity that permits one to characterize and classify hydrogen storage materials. Formation energy is defined as the difference between the sum of the total energies of the products and the reactants:

$$
\left.\Delta H=\sum E_{\text {tot }}(\text { products })-\sum E_{\text {tot }} \text { (reactants }\right)
$$

The formation of $\mathrm{MH}(\mathrm{M}=\mathrm{Li}, \mathrm{Na})$ materials from their constituting elements is given as:

$$
M+H \rightarrow M H
$$

The formation energy can be written as:

$$
\Delta H=E_{\text {tot }}(M H)-E_{\text {tot }}(M)-E_{\text {tot }}(H)
$$

The total energies of $\mathrm{LiH}, \mathrm{NaH}, \mathrm{Li}, \mathrm{Na}$, and $\mathrm{H}$ were calculated. The formation energies of $\mathrm{LiH}$ and $\mathrm{NaH}$ were found to be $-88.91 \mathrm{~kJ} . \mathrm{molH}_{2}$ and $-109.054 \mathrm{~kJ} . \mathrm{molH}_{2}$, respectively. The desorption temperature of hydrides was estimated using the standard Gibbs energy:

$$
\Delta G=\Delta H-T \Delta S
$$

where $\Delta H$ and $\Delta S$ are the enthalpy of formation and the standard entropy of the reaction respectively. At equilibrium, the standard Gibbs energy $\Delta G$ is zero. Therefore, the desorption temperature can be expressed as:

$$
T=-\frac{\Delta H}{\Delta S}
$$

The calculated desorption temperature was $680.33 \mathrm{~K}$ for $\mathrm{LiH}$ and $834.38 \mathrm{~K}$ for $\mathrm{NaH}$. The gravimetric hydrogen storage capacity can be determined as:

$$
C_{w t \%}=\left(\frac{\left(\frac{H}{M}\right) M_{H}}{M_{\text {Host }}+\left(\frac{H}{M}\right) M_{H}} \times 100\right)
$$

where $H / M$ is the hydrogen to material atom ratio, $M_{H}$ is the molar mass of $H$, and $M_{\text {Host }}$ is the molar mass of the host material. The hydrogen storage capacities calculated for $\mathrm{LiH}$ and $\mathrm{NaH}$ were $22.5 \%$ and $8.06 \%$, respectively.

(a)

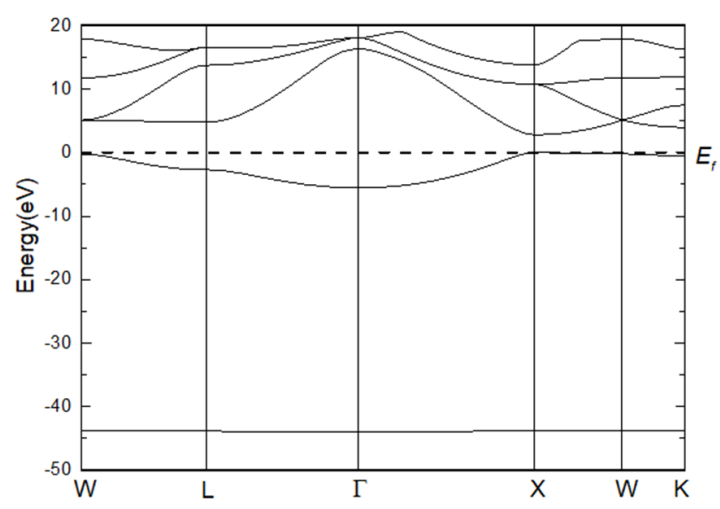

(b)

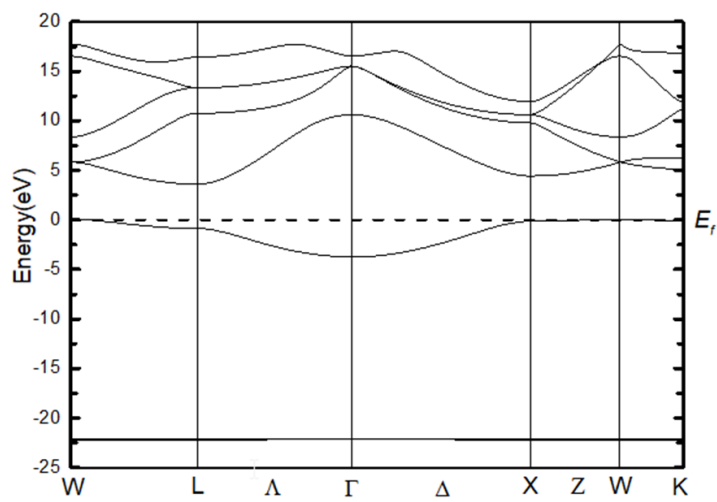

Fig. 1. Electron band structure calculated using TB-mBJ-GGA along high symmetry directions for (a) $\mathrm{LiH}$ and (b) $\mathrm{NaH}$. 
The electronic and optical properties of the materials are classically described by their electronic band structure [30-33]. Accurate knowledge of the band parameters of a given material provides precious information regarding its synthesis and devices fabrication [34-37]. The results of using the GGA$\mathrm{TBmBJ}$ approach to compute the electronic band structure of rocksalt $\mathrm{LiH}$ and $\mathrm{NaH}$ are plotted in Figure 1. It should be noted that, from a qualitative point of view, the same picture is observed for all the reported band structures. The Fermi level energy does not cross any energy bands for all questioned crystal materials. Therefore, $\mathrm{LiH}$ and $\mathrm{NaH}$ in the rocksalt structure can be considered respectively as semiconductor and insulating materials. According to Figure 1, the valence band maxima and the conduction band minima are located at the same high symmetry point $\mathrm{X}$ in the Brillouin zone. The band gaps of these materials are direct in nature, agreeing with the results of [22]. The fundamental difference between the band structures arises from the magnitude of their fundamental energy band gap, which differs from one band structure to another. The calculated fundamental direct band gap (X-X) for the rocksalt $\mathrm{LiH}$ and $\mathrm{NaH}$ were 3.05 and $3.65 \mathrm{eV}$ respectively.

(a)

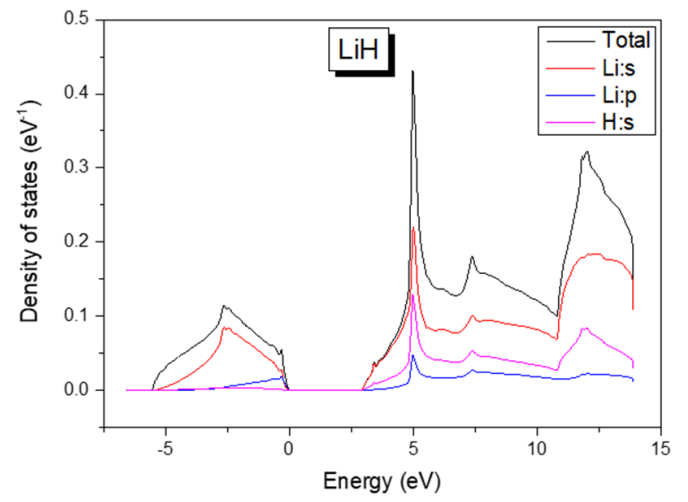

(b)

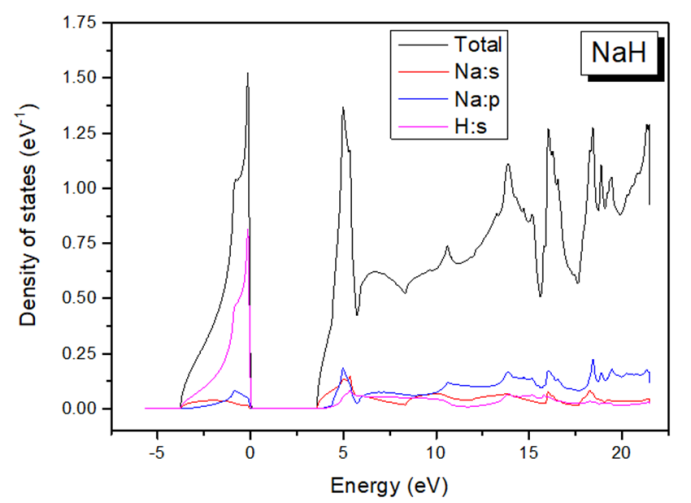

Fig. 2. Density of states (DOS) calculated using TB-mBJ-GGA for (a) $\mathrm{LiH}$ and (b) $\mathrm{NaH}$.

The properties of semiconductors can be related to the electron configuration shown by the molecules. The Densities Of States (DOS), total and projected, were computed for the rocksalt $\mathrm{LiH}$ and $\mathrm{NaH}$ using the TB-mBJ approach to show how the electrons are distributed on different orbitals. The results are shown in Figure 2. An inspection of $\mathrm{LiH}$ (Figure 2(a)) shows that the first region of the DOS is the most tightly bound energy band. The contribution of the Li-s electrons is predominant for the energy bands near the Fermi level of the $\mathrm{LiH}$ crystal. Hence, the Li-s electrons are useful for the conductibility of the crystal of interest. The DOS at these regions consist essentially of Li-s and Li-p states. The contribution of the H-s states was remarkably weak. Figure 2a also shows that the bonding peaks ranged from about -5 to $0 \mathrm{eV}$. These peaks were mainly formed by the Li-s state. For $\mathrm{NaH}$ (Figure 2b), it is observed that the intensity of the H-s orbitals in the valence band constitutes the dominant contribution to the DOS. The Na-p and Na-s states have a minor contribution to the DOS compared to the H-s orbitals. The bonding peaks ranged from about -3.75 to $0 \mathrm{eV}$. Moreover, the optical properties, such as the dielectric function, were examined. The computed optical response functions, real $\varepsilon_{l}(E)$ and imaginary $\varepsilon_{2}(E)$, for $\mathrm{LiH}$ and $\mathrm{NaH}$ are displayed in Figures 3 and 4 respectively, where $E$ is the photon energy. The main peak of the real parts of $\mathrm{LiH}$ and $\mathrm{NaH}$ occurs around $E=2.94 \mathrm{eV}$ and $E=4.58 \mathrm{eV}$ respectively.

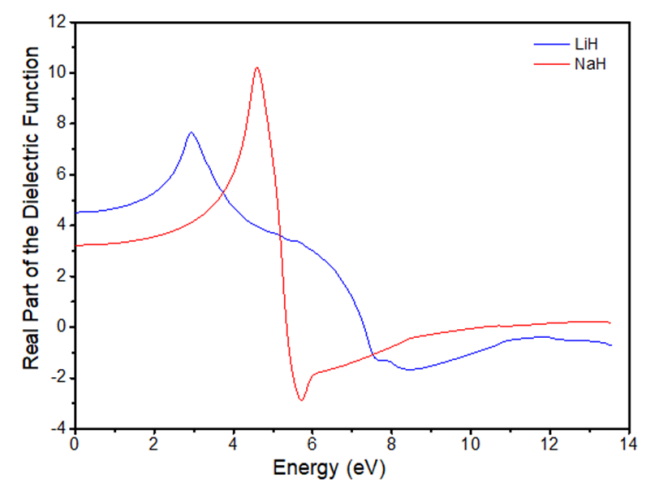

Fig. 3. Real part of the dielectric function calculated using TB-mBJ-GGA.

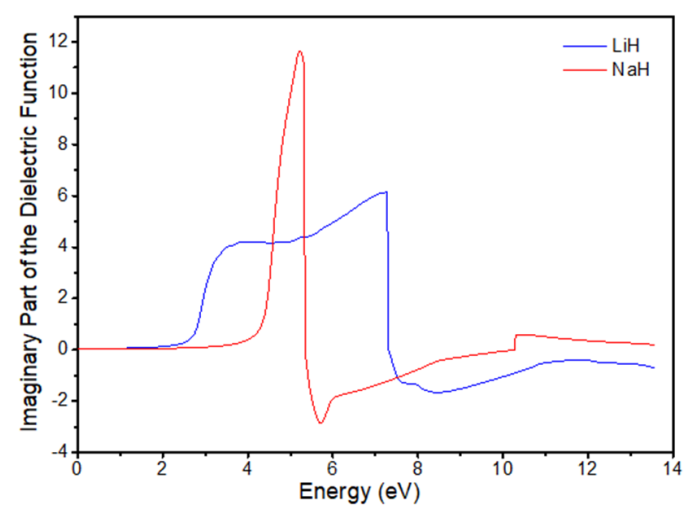

Fig. 4. Imaginary part of the dielectric function calculated using TB-mBJGGA.

Based on the optical transition mechanisms, the optical spectrum of a given crystal is usually classified into several photon energy regions [38]. In general, three spectral regions are distinguished. The first one is known as the reststrahlen region and is characterized by the interactions between the radiation field and the fundamental lattice vibrations [38]. For very low energies, the real part of the dielectric function tends to the static or low-energy dielectric constant $\varepsilon_{0}$. The $\varepsilon_{0}$ for $\mathrm{LiH}$ and $\mathrm{NaH}$ materials were determined as: 


$$
\begin{gathered}
\varepsilon_{0}(L i H)=4.55 \\
\varepsilon_{0}(N a H)=3.21
\end{gathered}
$$

Regarding the $\varepsilon_{0}$ of $\mathrm{LiH}$, this result is different from the quoted in [39]. To the best of our knowledge, no data regarding $\varepsilon_{0}$ for $\mathrm{NaH}$ has been reported so far. Thus, this result could serve as a reference. The optical constant that relates the socalled reststrahlen region to the near-infrared region is commonly known as the high-frequency dielectric constant $\varepsilon_{\infty}$. For these materials, the calculations gave:

$$
\begin{gathered}
\varepsilon_{\infty}(L i H)=7.84 \\
\varepsilon_{\infty}(N a H)=10.82
\end{gathered}
$$

The calculated $\varepsilon_{\infty}$ for $\mathrm{LiH}$ shows reasonable agreement with the one reported in [34]. For $\mathrm{NaH}$, the calculation in [40] gave a value of 3.71 , which is too low compared to this result. The general shape of $\varepsilon_{1}$ was expected for a harmonic oscillator with resonant frequencies of about 8.39 and $5.71 \mathrm{eV}$ for $\mathrm{LiH}$ and $\mathrm{NaH}$, respectively. It is generally accepted that the resonance frequency is a fundamental property of a given material. This quantity allows the expression of the average energy that separates the levels of bonding and anti-bonding [30]. The absorption for the imaginary part of the dielectric function starts at about 2.04 and $3.09 \mathrm{eV}$ for $\mathrm{LiH}$ and $\mathrm{NaH}$, respectively. Similar behavior was reported in $[41,42]$ for $\mathrm{ZnTe}_{1-\mathrm{x}} \mathrm{O}_{\mathrm{x}}$ alloys. Knowledge of the refractive index of semiconductors in the energy range below or near the fundamental absorption edge is mostly interesting in device design [43-48]. This refractive device $(n)$ can be easily derived from the dielectric function $\varepsilon(E)$. The $n$ values obtained for $\mathrm{LiH}$ and $\mathrm{NaH}$ were:

$$
\begin{gathered}
n(\mathrm{LiH})=2.8 \\
n(\mathrm{NaH})=3.29
\end{gathered}
$$

The $n$ value for $\mathrm{LiH}$ is in reasonable agreement with the value of 1.47 reported in [49]. The calculated spectral dependence of $n(E)$ for $\mathrm{LiH}$ and $\mathrm{NaH}$ is shown in Figure 5.

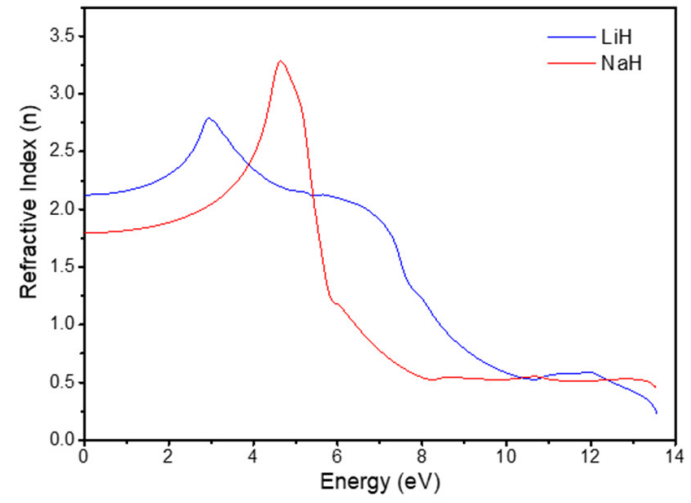

Fig. 5. Refractive index calculated using TB-mBJ-GGA for LiH and $\mathrm{NaH}$.

From this spectrum, it can be noted that clear peaks are present. These peaks are due to the excitonic transitions at the $E_{0}$ edges. Excitonic effects are believed to be responsible for the enhancement of the oscillator strength at the critical points $M_{0}$ and $M_{1}$ [50]. Since the total strength of the oscillator is proportional to the number of valence electrons, it should be "conserved" in some way. This means that as the oscillator strength at the critical points $M_{0}$ and $M I$ is enhanced due to excitonic interactions, some losses occur elsewhere to achieve strength conservation. The strongest peaks in the $n(E)$ spectra can be explained as the result of the $2 \mathrm{D}$ exciton transition $\left(E_{1}\right)$.

\section{CONCLUSION}

This study examined the structural, electronic, and optical properties of the alkali metal hydrides $\mathrm{LiH}$ and $\mathrm{NaH}$, using the FP-LAPW technique within DFT in the WC-GGA for structural properties and TB-mBJ-GGA for electronic and optical features. The crystal structure of both investigated materials investigated was rocksalt (B1). Results regarding the lattice constant, bulk modulus, and pressure derivative for $\mathrm{LiH}$ and $\mathrm{NaH}$ were reported and compared, where possible, with the available experimental and theoretical data. In general, a reasonably good agreement was found between the results of this study and the literature. The electron band structure, the density of states, and the dependence of the real and imaginary parts of the dielectric function along the refractive index of the materials under load were also studied and analyzed. The results derived from the present study could be useful for hydrogen storage purposes.

\section{REFERENCES}

[1] L. Schlapbach and A. Züttel, "Hydrogen-storage materials for mobile applications," Nature, vol. 414, no. 6861, pp. 353-358, Nov. 2001, https://doi.org/10.1038/35104634.

[2] A. Léon, Hydrogen Technology: Mobile and Portable Applications. Berlin-Heidelberg, Germany: Springer Science \& Business Media, 2008.

[3] S. Javadpoor and D. Nazarpour, "Modeling a PV-FC-Hydrogen Hybrid Power Generation System," Engineering, Technology \& Applied Science Research, vol. 7, no. 2, pp. 1455-1459, Apr. 2017, https://doi.org/ 10.48084/etasr.760.

[4] M. N. Kordkandy, A. Arash, and M. N. Kordkandy, "Hydrogen Gas Production in a Stand-Alone Wind Farm," Engineering, Technology \& Applied Science Research, vol. 7, no. 2, pp. 1444-1449, Apr. 2017, https://doi.org/10.48084/etasr.991.

[5] A. H. El-Sinawi, "Production of Hydrogen from Poly Ethylene Terephthalate (PET) using CT 434 ZSM-5 Catalyst at Considerably Low Temperatures," Engineering, Technology \& Applied Science Research, vol. 6, no. 6, pp. 1269-1273, Dec. 2016, https://doi.org/10.48084/ etasr.822.

[6] R. S. Irani, "Hydrogen Storage: High-Pressure Gas Containment," MRS Bulletin, vol. 27, no. 9, pp. 680-682, Sep. 2002, https://doi.org/ $10.1557 / \mathrm{mrs} 2002.221$.

[7] V. A. Yartys and M. V. Lototsky, "An Overview of Hydrogen Storage Methods," in Hydrogen Materials Science and Chemistry of Carbon Nanomaterials, Dordrecht, Netherlands, 2005, pp. 75-104, https://doi.org/10.1007/1-4020-2669-2_7.

[8] U. Eberle, M. Felderhoff, and F. Schüth, "Chemical and Physical Solutions for Hydrogen Storage," Angewandte Chemie International Edition, vol. 48, no. 36, pp. 6608-6630, Aug. 2009, https://doi.org/ 10.1002/anie.200806293.

[9] J. Wolf, "Liquid-Hydrogen Technology for Vehicles," MRS Bulletin, vol. 27, no. 9, pp. 684-687, Sep. 2002, https://doi.org/10.1557/mrs2002. 222.

[10] N. T. Stetson and L. S. Blair, "Hydrogen Storage Technologies - A Tutorial with Perspectives from the US National Program," in Materials Challenges in Alternative and Renewable Energy, Hoboken, NJ, USA: John Wiley \& Sons, 2012. 
[11] "Definitions, Terminology, and Symbols in Colloid and Surface Chemistry," Pure and Applied Chemistry, vol. 31, no. 4, pp. 579-638, 1972.

[12] J. Yang, A. Sudik, C. Wolverton, and D. J. Siegel, "High capacity hydrogen storage materials: attributes for automotive applications and techniques for materials discovery," Chemical Society Reviews, vol. 39, no. 2, pp. 656-675, Jan. 2010, https://doi.org/10.1039/B802882F.

[13] P. Hohenberg and W. Kohn, "Inhomogeneous Electron Gas," Physical Review, vol. 136, no. 3B, pp. B864-B871, Nov. 1964, https://doi.org/ 10.1103/PhysRev.136.B864.

[14] P. Blaha et al., WIEN2k - An Augmented Plane Wave + Local Orbitals Program for Calculating Crystal Properties. Vienna, Austria: Vienna University of Technology, 2021.

[15] Z. Wu and R. E. Cohen, "More accurate generalized gradient approximation for solids," Physical Review B, vol. 73, no. 23, Jun. 2006, Art. no. 235116, https://doi.org/10.1103/PhysRevB.73.235116.

[16] F. Tran and P. Blaha, "Accurate Band Gaps of Semiconductors and Insulators with a Semilocal Exchange-Correlation Potential," Physical Review Letters, vol. 102, no. 22, Jun. 2009, Art. no. 226401, https://doi.org/10.1103/PhysRevLett.102.226401.

[17] H. J. Monkhorst and J. D. Pack, "Special points for Brillouin-zone integrations," Physical Review B, vol. 13, no. 12, pp. 5188-5192, Jun. 1976, https://doi.org/10.1103/PhysRevB.13.5188.

[18] A. Gueddim, N. Fakroun, N. Bouarissa, and A. Villesuzanne, "A density functional study of structural and elastic properties of LaN under high pressure," Materials Chemistry and Physics, vol. 118, no. 2, pp. 427431, Dec. 2009, https://doi.org/10.1016/j.matchemphys.2009.08.012.

[19] A. Bellouche, A. Gueddim, S. Zerroug, and N. Bouarissa, "Elastic properties and optical spectra of $\mathrm{ZnS}_{1-\mathrm{x}} \mathrm{O}_{\mathrm{x}}$ dilute semiconductor alloys," Optik, vol. 127, no. 23, pp. 11374-11378, Dec. 2016, https://doi.org/ 10.1016/j.ijleo.2016.09.034.

[20] A. Gueddim, M. E. Madjet, S. Zerroug, and N. Bouarissa, "Firstprinciples investigations of electronic properties and optical spectra of $\mathrm{Cd}_{1-\mathrm{x}} \mathrm{Mn}_{\mathrm{x}} \mathrm{Te}$ dilute magnetic semiconductors," Optical and Quantum Electronics, vol. 48, no. 12, Nov. 2016, Art. no. 551, https://doi.org/ 10.1007/s11082-016-0818-1.

[21] F. D. Murnaghan, "The Compressibility of Media under Extreme Pressures," Proceedings of the National Academy of Sciences of the United States of America, vol. 30, no. 9, pp. 244-247, Sep. 1944.

[22] N. Settouti and H. Aourag, "Structural and mechanical properties of alkali hydrides investigated by the first-principles calculations and principal component analysis," Solid State Sciences, vol. 58, pp. 30-36, Aug. 2016, https://doi.org/10.1016/j.solidstatesciences.2016.05.006.

[23] J. Zhang et al., "Phonon and elastic instabilities in rocksalt alkali hydrides under pressure: First-principles study," Physical Review B, vol. 75, no. 10, Mar. 2007, Art. no. 104115, https://doi.org/10.1103/ PhysRevB.75.104115.

[24] J. Hama, K. Suito, and N. Kawakami, "First-principles calculation of the shock-wave equation of state of isotopic lithium hydrides," Physical Review B, vol. 39, no. 5, pp. 3351-3360, Feb. 1989, https://doi.org/ 10.1103/PhysRevB.39.3351.

[25] S. J. Duclos, Y. K. Vohra, A. L. Ruoff, S. Filipek, and B. Baranowski, "High-pressure studies of $\mathrm{NaH}$ to $54 \mathrm{GPa}$," Physical Review B, vol. 36, no. 14, pp. 7664-7667, Nov. 1987, https://doi.org/10.1103/PhysRevB. 36.7664.

[26] W. Yu, C. Jin, and A. Kohlmeyer, "First principles calculation of phonon dispersion, thermodynamic properties andB1-to-B2 phase transition of lighter alkali hydrides," Journal of Physics: Condensed Matter, vol. 19, no. 8, Feb. 2007, Art. no. 086209, https://doi.org/ $10.1088 / 0953-8984 / 19 / 8 / 086209$.

[27] A. Gueddim, S. Zerroug, and N. Bouarissa, "Composition dependence of the optical properties and band structure of the zinc-blende $\mathrm{ZnS}_{1-\mathrm{x}} \mathrm{O}_{\mathrm{x}}$ : a first principles study," Philosophical Magazine, vol. 95, no. 24, pp. 2627-2638, Aug. 2015, https://doi.org/10.1080/14786435.2015.1073 401 .

[28] S. Zerroug, A. Gueddim, and N. Bouarissa, "Composition dependence of fundamental properties of $\mathrm{Cd}_{1-\mathrm{x}} \mathrm{Co}_{\mathrm{x}} \mathrm{Te}$ magnetic semiconductor alloys,"
Journal of Computational Electronics, vol. 15, no. 2, pp. 473-478, Jun. 2016, https://doi.org/10.1007/s10825-016-0802-9.

[29] A. Gueddim, S. Zerroug, N. Bouarissa, and N. Fakroun, "Study of the elastic properties and wave velocities of rocksalt $\mathrm{Mg}_{1-\mathrm{x}} \mathrm{Fe}_{\mathrm{x}} \mathrm{O}$ : ab initio calculations," Chinese Journal of Physics, vol. 55, no. 4, pp. 1423-1431, Aug. 2017, https://doi.org/10.1016/j.cjph.2017.04.009.

[30] M. L. Cohen and J. R. Chelikowsky, Electronic Structure and Optical Properties of Semiconductors. Berlin-Heidelberg, Germany: SpringerVerlag, 1989.

[31] R. M. Martin, Electronic Structure: Basic Theory and Practical Methods. Cambridge, UK: Cambridge University Press, 2020.

[32] A. Gueddim, R. Zerdoum, and N. Bouarissa, "Dependence of electronic properties on nitrogen concentration in $\mathrm{GaAs}_{1-\mathrm{x}} \mathrm{N}_{\mathrm{x}}$ dilute alloys," Journal of Physics and Chemistry of Solids, vol. 67, no. 8, pp. 1618-1622, Aug. 2006, https://doi.org/10.1016/j.jpcs.2006.02.007.

[33] A. Gueddim and N. Bouarissa, "Electronic structure and optical properties of dilute $\operatorname{InAs}_{1-\mathrm{x}} \mathrm{N}_{\mathrm{x}}$ : pseudopotential calculations," Physica Scripta, vol. 80, no. 1, Jun. 2009, Art. no. 015701, https://doi.org/ 10.1088/0031-8949/80/01/015701.

[34] I. Vurgaftman, J. R. Meyer, and L. R. Ram-Mohan, "Band parameters for III-V compound semiconductors and their alloys," Journal of Applied Physics, vol. 89, no. 11, pp. 5815-5875, Jun. 2001, https://doi.org/10.1063/1.1368156.

[35] S. Adachi, Properties of Semiconductor Alloys: Group-IV, III-V and IIVI Semiconductors. Chichester, West Sussex, UK: John Wiley \& Sons, 2009.

[36] N. Bouarissa and F. Annane, "Electronic properties and elastic constants of the ordered $\mathrm{Ge}_{1-\mathrm{x}} \mathrm{Sn}_{\mathrm{x}}$ alloys," Materials Science and Engineering: B, vol. 95, no. 2, pp. 100-106, Aug. 2002, https://doi.org/10.1016/S09215107(02)00203-9.

[37] N. Bouarissa, "Electron and Positron Energy Levels and Deformation Potentials in Group-III Nitrides," Physica Status Solidi (B), vol. 231, no. 2, pp. 391-402, 2002, https://doi.org/10.1002/1521-3951(200206)231: 2<391::AID-PSSB391>3.0.CO;2-J.

[38] S. Adachi, Properties of Group-IV, III-V and II-VI Semiconductors. Chichester, West Sussex, UK: John Wiley \& Sons, Ltd, 2005.

[39] F. E. Pretzel, G. N. Rupert, C. L. Mader, E. K. Storms, G. V. Gritton, and C. C. Rushing, "Properties of lithium hydride I. Single crystals," Journal of Physics and Chemistry of Solids, vol. 16, no. 1, pp. 10-20, Nov. 1960, https://doi.org/10.1016/0022-3697(60)90064-0.

[40] D. K. Blat, N. E. Zein, and V. I. Zinenko, "Calculations of phonon frequencies and dielectric constants of alkali hydrides via the density functional method," Journal of Physics: Condensed Matter, vol. 3, no. 29, pp. 5515-5524, Jul. 1991, https://doi.org/10.1088/0953-8984/ $3 / 29 / 006$.

[41] S. Zerroug, A. Gueddim, M. Ajmal Khan, and N. Bouarissa, "Ab initio study of structural parameters and optical properties of $\mathrm{ZnTe}_{1-\mathrm{x}} \mathrm{O}_{\mathrm{x}}$," Superlattices and Microstructures, vol. 53, pp. 155-162, Jan. 2013, https://doi.org/10.1016/j.spmi.2012.09.015.

[42] A. Gueddim, S. Zerroug, and N. Bouarissa, "Optical characteristics of $\mathrm{ZnTe} 1-\mathrm{xOx}$ alloys from first-principles calculations," Journal of Luminescence, vol. 135, pp. 243-247, Mar. 2013, https://doi.org/ 10.1016/j.jlumin.2012.10.004.

[43] S. Ozaki and S. Adachi, "Optical constants of $\mathrm{ZnS}_{\mathrm{x}} \mathrm{Se}_{1-\mathrm{x}}$ ternary alloys," Journal of Applied Physics, vol. 75, no. 11, pp. 7470-7475, Jun. 1994, https://doi.org/10.1063/1.356617.

[44] S. Ozaki and S. Adachi, "Optical constants of $\mathrm{ZnS}_{\mathrm{x}} \mathrm{Se}_{1-\mathrm{x}}$ ternary alloys," Journal of Applied Physics, vol. 75, no. 11, pp. 7470-7475, Jun. 1994, https://doi.org/10.1063/1.356617.

[45] A. Gueddim and N. Bouarissa, "Theoretical investigation of the conduction and valence band offsets of $\mathrm{GaAs}_{1-\mathrm{x}} \mathrm{N}_{\mathrm{x}} / \mathrm{GaAs}_{1-\mathrm{y}} \mathrm{N}_{\mathrm{y}}$ heterointerfaces," Applied Surface Science, vol. 253, no. 17, pp. 73367341, Jun. 2007, https://doi.org/10.1016/j.apsusc.2007.03.019.

[46] A. Gueddim, R. Zerdoum, and N. Bouarissa, "Effect of nitrogen concentration on mechanical properties of $\mathrm{GaAs}_{1-\mathrm{x}} \mathrm{N}_{\mathrm{x}}$ dilute alloys," Materials Science and Engineering: B, vol. 131, no. 1, pp. 111-115, Jul. 2006, https://doi.org/10.1016/j.mseb.2006.03.032. 
[47] P. Hervé and L. K. J. Vandamme, "General relation between refractive index and energy gap in semiconductors," Infrared Physics \& Technology, vol. 35, no. 4, pp. 609-615, Jun. 1994, https://doi.org/ 10.1016/1350-4495(94)90026-4.

[48] N. M. Ravindra, P. Ganapathy, and J. Choi, "Energy gap-refractive index relations in semiconductors - An overview," Infrared Physics \& Technology, vol. 50, no. 1, pp. 21-29, Mar. 2007, https://doi.org/ 10.1016/j.infrared.2006.04.001.

[49] R. C. Weast, Handbook of Chemistry and Physics. Boca Ration, FL, USA: John Wiley \& Sons, 1972.

[50] P. Y. Yu and M. Cardona, Fundamentals of Semiconductors. Berlin, Heidelberg, Germany: Springer, 2010. 